\title{
Immunotherapy of lung cancer
}

Strategies to modulate the body's innate and adaptive immunity to treat advanced stage cancer has taken central stage in the management of solid and hematologic malignancies. Agents targeting the programmed death-1 (PD-1) pathway in particular, have become established therapies for various types of cancer. In the last five years, clinical and translational research efforts have made immunotherapy a cornerstone of therapeutic interventions for advanced stage non-small cell lung cancer (NSCLC) leading to approval of atezolizumab, nivolumab and pembrolizumab for previously treated NSCLC. Pembrolizumab is also approved as a single agent and in combination with doublet chemotherapy for newly diagnosed non-squamous NSCLC. Treatment with durvalumab, based on recent reports, has led to improved outcome for patients with locally advanced NSCLC, regardless of histology or level of programmed death-ligand 1 (PD-L1) expression. The series of review articles in this issue of Fournal of Thoracic Diseases (FTD) represents a systematic overview of the progress made to date in the effort to incorporate immunotherapy into lung cancer management. The review articles address various important aspects including potential overhype of immunotherapy benefit as a result of repeated presentations of the same immunotherapy trial results at professional meetings and the established role of immunotherapy in frontline and post frontline therapy of NSCLC. Additionally, our guest authors contextualized the promising results of immunotherapy clinical trials in the neoadjuvant setting for early stage NSCLC and of combined anti PD-1 and anti CTLA-4 immunotherapy for SCLC. Emerging strategies to further enhance benefit of immunotherapy through combination with cytotoxic agent, immune targeted agents and radiation therapy are also addressed. Despite the overall safety profile of immunotherapy, these agents have unique toxicities. The optimal management of immune-mediated toxicities are also discussed in this special issue. We hope you find this special edition of the journal informative and we expect that it will be a dependable reference on the present status and future trajectory of immune-based therapy of lung cancer for many years to come.

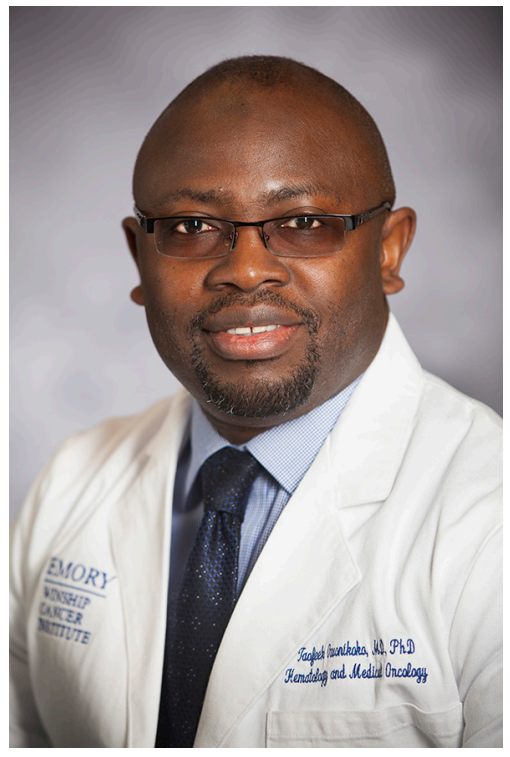

Taofeek K. Owonikoko

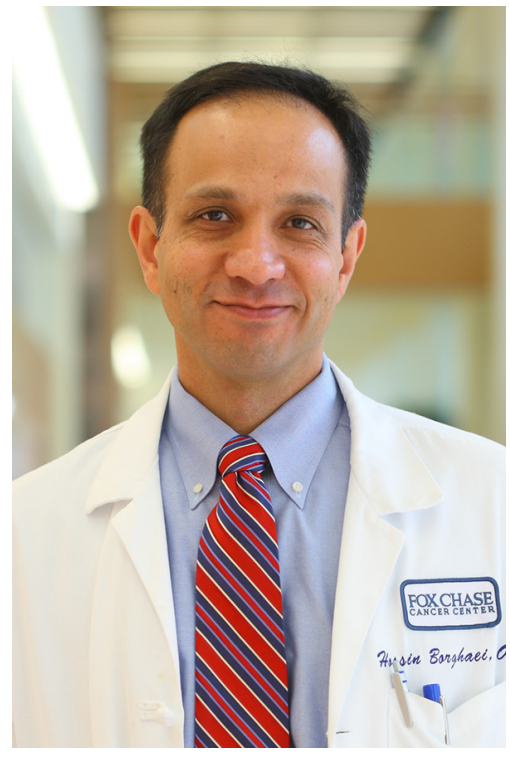

Hossein Borghaei 


\section{Acknowledgements}

None.

Taofeek K. Owonikoko, MD, PhD

Guest Editor, Professor and Vice Chair, Co-Leader of Thoracic Oncology, Department of Hematology \& Medical Oncology, Winship Cancer Institute of Emory University, Atlanta, GA, USA. (Email: towonik@emory.edu)

Hossein Borghaei, MS, DO Associate Guest Editor, Associate Professor, Chief, Thoracic Oncology, Department of Medicine, Fox Chase Cancer Center/Temple University, Philadelphia, PA, USA. (Email: Hossein.Borghaei@fccc.edu) doi: $10.21037 /$ jtd.2018.01.142 Conflicts of Interest: The authors have no conflicts of interest to declare. View this article at: http://dx.doi.org/10.21037/jtd.2018.01.142

Cite this article as: Owonikoko TK, Borghaei $\mathrm{H}$. Immunotherapy of lung cancer. J Thorac Dis 2018;10(Suppl 3):S395-S396. doi: 10.21037/jtd.2018.01.142 